Sherwin DeSouza, MD

Department of Hematology/Oncology,

University Hospitals Seidman Cancer Center,

Case Comprehensive Cancer Center, Cleveland, $\mathrm{OH}$
Dana Angelini, MD

Associate Staff, Department of Hematology

and Medical Oncology, Taussig Cancer Institute,

Cleveland Clinic, Cleveland, $\mathrm{OH}$

\title{
Updated guidelines for immune thrombocytopenic purpura: Expanded management options
}

\section{ABSTRACT}

The current American Society of Hematology (ASH) guidelines for the management of patients with immune thrombocytopenic purpura (ITP) are an update to the 2011 guidelines. The updates focus on treating patients with ITP without bleeding in both outpatient and inpatient settings, including those with newly diagnosed, persistent, and chronic ITP refractory to first-line therapy. Recommendations for therapy include corticosteroids, intravenous immunoglobulins, anti-D immunoglobulin, rituximab, splenectomy, and thrombopoietin-receptor agonists, as well as observation.

\section{KEY POINTS}

Inpatient management is suggested for patients with newly diagnosed ITP who have a platelet count below 20 $\times 10^{9} / \mathrm{L}$ and are asymptomatic or have minor symptoms.

Outpatient management can be considered in patients with a platelet count of at least $20 \times 10^{9} / \mathrm{L}$ who are asymptomatic or have minor mucocutaneous bleeding.

Observation can be considered for newly diagnosed patients with a platelet count of at least $30 \times 10^{9} / \mathrm{L}$ who are asymptomatic or have minor mucocutaneous bleeding.

Corticosteroid therapy should be considered for newly diagnosed patients with a platelet count less than $30 \times$ $10^{9} / \mathrm{L}$ who are asymptomatic, or for patients with minor or more significant bleeding.
T MMUNE THROMBOCYTOPENIC PURPURA (ITP) is an acquired autoimmune disorder characterized by thrombocytopenia caused by autoantibodies against platelet antigens. ITP is a diagnosis of exclusion, with an estimated incidence of 2 to 5 per 100,000 people in the general population. ${ }^{1}$

The updated American Society of Hematology (ASH) guidelines for the management of patients with ITP, published in 2019, ${ }^{1}$ are based on systematic reviews that included hundreds of studies by a multidisciplinary panel under the direction of the University of Oklahoma Health Sciences Center. Recommendations cover management strategies for ITP in patients with newly diagnosed, persistent, and refractory disease and include therapy with corticosteroids, intravenous (IV) immunoglobulins, anti-D immunoglobulins, rituximab, splenectomy, and thrombopoietin receptor agonists, as well as observation.

\section{WHAT'S NEW IN THE GUIDELINES?}

The main focus of the guidelines is on patients with ITP without bleeding in both outpatient and inpatient settings. The purpose is to help practitioners decide on inpatient vs outpatient management, thresholds for when to initiate treatment, and options for second-line treatment in adults. Pediatric patients are discussed in the guidelines, but that population is beyond the scope of this review. Table 1 lists the key differences between the 2019 update and the previous guidelines for the management of patients with ITP. 1,2 


\section{TABLE 1}

\section{Current vs previous guidelines on immune thrombocytopenic purpura in adults}

\begin{tabular}{ll}
\hline Nomenclature & \multicolumn{1}{c}{2019} \\
\hline Diagnosis & Corticosteroid dependence recognized as an entity needing \\
& Diagnosis of ITP not discussed \\
Criteria for admission & $\begin{array}{l}\text { Inpatient vs outpatient } \\
\text { Inpatient: Platelet count }<20 \times 10^{9} / \mathrm{L} \text { asymptomatic } \\
\text { or minor symptoms and new diagnosis } \\
\text { Outpatient: Platelet count } \geq 20 \times 10^{9} / \mathrm{L} \text { asymptomatic or } \\
\text { minor symptoms or established ITP }\end{array}$ \\
& $\begin{array}{l}\text { Choice of agent } \\
\text { Eirst-line therapy } \\
\text { (40 mg/day for } 4 \text { days); dexamethasone preferred if rapidity } \\
\text { of response is valued } \\
\text { Corticosteroids alone vs in combination. Prefer corti- } \\
\text { costeroids alone rather than in combination with rituximab } \\
\text { for initial treatment } \\
\text { Duration of therapy } \\
\text { Recommends in favor of short course ( } \leq 6 \text { weeks) and } \\
\text { against longer course of prednisone ( }>6 \text { weeks including } \\
\text { taper) }\end{array}$ \\
\hline
\end{tabular}

Second-line therapy

Special populations and other considerations
Introduces concept of shared decision-making with patients, particularly with regard to the choice of secondline therapy

Provides guidance on considerations while choosing second-line therapy

Elderly

Raises concern regarding potential complications of steroid use in elderly and those with diabetes

Cost

Considers eltrombopag more cost-effective than romiplostim

Rituximab and splenectomy are considered cost-equivalent, but TPO-RAs are more expensive and may not be covered by all insurance payers
Workup including HIV, hepatitis C testing, and bone marrow biopsy discussed

Inpatient vs outpatient not discussed

\section{Choice of agent}

Anti-D immunoglobulins added as a treatment option for Rh-positive, nonsplenectomized patients

\section{Duration of therapy}

Longer course of steroid (prednisone $1 \mathrm{mg} / \mathrm{kg} \times 21$ days followed by taper) recommended over shorter course

\section{Choice of therapy}

Splenectomy if steroids fail

TPO-RA for relapse after splenectomy or if splenectomy is contraindicated

Rituximab after failure of steroids, IVIG, or splenectomy

Discusses management of ITP in pregnancy and treatment of secondary ITP

HIV = human immunodeficiency virus; ITP = immune thrombocytopenic purpura; IVIG = intravenous immune globulin; TPO-RA = thrombopoietin-receptor agonist 


\section{Inpatient vs outpatient management}

Inpatient management is suggested for those with newly diagnosed ITP who have a platelet count below $20 \times 10^{9} / \mathrm{L}$ and are asymptomatic or have minor symptoms such as wet purpura, gum bleeding, continuous epistaxis needing intervention, menorrhagia, or multiple large bruises larger than $3 \mathrm{~cm}$.

Outpatient management can be considered for patients with a platelet count of at least $20 \times 10^{9} / \mathrm{L}$ who are asymptomatic or have minor mucocutaneous bleeding such as few petechiae, small bruises of less than $3 \mathrm{~cm}$, or epistaxis on nose-blowing. It can also be considered for patients with established ITP who have a platelet count below $20 \times 10^{9} / \mathrm{L}$ and are asymptomatic or have minor symptoms. Asymptomatic patients or those with a documented good response to rescue agents can be followed as outpatients.

\section{Observation vs corticosteroid therapy}

Observation can be considered for newly diagnosed patients with a platelet count of at least $30 \times 10^{9} / \mathrm{L}$ who are asymptomatic or have minor mucocutaneous bleeding. The updated guidelines note the need for clinical judgment

The guidelines

focus on

inpatient

and outpatient

treatment

for patients

with ITP

without

bleeding for patients who have additional comorbidities, who are scheduled for procedures, or who have more than minor bleeding. It is important to consider concomitant medications such as anticoagulant and antiplatelet drugs, as higher platelet thresholds are more desirable in this setting. Patients with a history of bleeding also warrant consideration for a higher platelet goal and may warrant treatment rather than observation.

Corticosteroid therapy should be considered for newly diagnosed patients with a platelet count less than $30 \times 10^{9} / \mathrm{L}$ who are asymptomatic or patients with minor or more significant bleeding. The presence of severe thrombocytopenia also warrants consideration for a more aggressive approach, such as a combination of high-dose steroids and other rescue agents (eg, IV immunoglobulins or anti-D immune globulins).

Steroid therapy warrants extra consideration in patients with poorly controlled diabetes and those who are immunocompromised.

\section{First-line therapy recommendations}

Regarding the type and duration of steroids, the guidelines recommend against a long course of prednisone ( $>6$ weeks including taper) in favor of a shorter course ( $\leq 6$ weeks). When considering dexamethasone vs prednisone, either is acceptable (prednisone $0.5-2.0 \mathrm{mg} / \mathrm{kg} /$ day or dexamethasone $40 \mathrm{mg} /$ day for 4 days). However, if a rapid response is desired, dexamethasone is preferred. Of note, there appears to be no benefit with regard to response at 1 month, durability of response, or major bleeding between these treatment options. Furthermore, practitioners should ensure that the patient is adequately monitored for potential corticosteroid side effects regardless of the duration or type of corticosteroid selected.

In addition, the guidelines suggest using corticosteroids alone for initial treatment rather than in combination with rituximab as first-line therapy.

\section{Second-line therapy recommendations}

The guidelines provide recommendations on managing adults with ITP who are corticosteroid-dependent or unresponsive to corticosteroids. Of note, corticosteroid dependence has been defined as an ongoing need for continuous prednisone at more than $5 \mathrm{mg} /$ day (or corticosteroid equivalent) or as requiring frequent courses of corticosteroids to maintain a platelet count of at least $30 \times 10^{9} / \mathrm{L}$ or to avoid bleeding.

The updated guidelines are based on retrospective and indirect comparisons given the lack of prospective clinical trial data from headto-head comparisons of second-line treatment options. Although the guidelines read as direct suggestions, in practice the recommendations for second-line therapy are based on shared decision-making after a review of risks and benefits and patient preferences.

When choosing a second-line therapy in adults with ITP lasting 3 months or longer, the guidelines suggest the following:

- Either splenectomy or a thrombopoietinreceptor agonist (TPO-RA), such as romiplostim or eltrombopag

- Rituximab rather than splenectomy

- A TPO-RA rather than rituximab.

When choosing between a TPO-RA, splenectomy, or rituximab for a second-line therapy, practitioners should use shared decisionmaking with the patient, taking into account 
patient preferences with regard to potential complications, side effects, and treatment duration, along with the following:

- If possible, splenectomy should be avoided within the first year of ITP diagnosis, given the potential for spontaneous remission

- If durability of response is valued, TPORAs or splenectomy can be considered over rituximab

- If avoidance of long-term medications is valued, rituximab or splenectomy may be considered over a TPO-RA agent

- If avoidance of surgery is the goal, rituximab or a TPO-RA may be preferred, recognizing that the latter option often requires a prolonged treatment course

- Patients should have appropriate immunizations before and after splenectomy

- Practitioners should educate the patient on prompt recognition and management of fever and refer to current recommendations on pre- and post-splenectomy care

- Infections can occur after treatment with rituximab, and hepatitis testing should be done before initiating rituximab.

For the TPO-RAs eltrombopag and romiplostim, it should be noted that no clinical trials have been completed that directly compared these agents. Guidelines suggest either eltrombopag or romiplostim, noting that individual patients may place a higher value on a daily oral medication vs weekly subcutaneous injection.

Of note, these guidelines did not mention avatrombopag as an option for a second-line agent. Avatrombopag is a TPO-RA approved by the US Food and Drug Administration (FDA) in 2019 for treating thrombocytopenia in patients with chronic ITP who have had an insufficient response to previous therapy. Avatrombopag is now considered an option for second-line therapy based on its FDA approval as well as safety and efficacy data showing that it is an effective option for patients with ITP who have had insufficient response to the initial treatment regimen.

Eltrombopag is considered more costeffective than romiplostim. Oral administration (eltrombopag and avatrombopag) vs subcutaneous injection (romiplostim) along with food interactions (with eltrombopag) should be discussed with the patient.

\section{DO OTHER SOCIETIES AGREE OR DISAGREE?}

A Joint Working Group representing several European hematological societies published guidelines in 2018. ${ }^{3}$ Their guidelines discuss treatment of patients with platelet counts below $20 \times 10^{9} / \mathrm{L}$ and observation for patients with higher platelet counts. There was agreement on generally shorter duration of steroids, but no preferred steroid was recommended. The use of rituximab and TPO-RAs is suggested as rescue therapy to raise platelet counts in the setting of severe hemorrhage in patients without adequate response to steroid or IV immune globulin therapy. However, for second-line therapy, TPO-RAs are favored over rituximab or splenectomy, and rituximab is recommended as third-line therapy after failure of TPO-RAs.

A dose-tapering regimen for eltrombopag or romiplostim is suggested for patients maintaining platelet counts above $50 \times 10^{9} / \mathrm{L}$ for several months. The use of a recombinant thrombopoietin molecule approved in China was discussed, noting particularly that it appears to be safe for use during pregnancy. Splenectomy is reserved as a last- resort therapy for patients failing all other lines of therapy, with a recommendation to reserve it until after the first 12 months of ITP treatment. The differences in guidelines are likely in part due to cost and economics and healthcare litigation concerns in the United States.

\section{WHAT IS THE CLINICAL IMPACT?}

For patients with a predictable response to rescue therapy, the updated ASH guidelines will help reduce hospital admissions for patients with asymptomatic ITP with severe thrombocytopenia. The advantages and disadvantages of available second-line therapies are briefly discussed to inform shared decision-making with patients. The guidelines stress the importance of monitoring for side effects of glucocorticoid therapy and highlight pre- and postsplenectomy vaccination care. Thus, the side effects of ITP treatment may be managed better by these guidelines. With multiple drugs approved for ITP management since the 2011 guidelines, the updated guidelines help to stratify the sequence of use of the newer drugs

\section{Guidelines recommend against a long course of prednisone ( $>6$ weeks including taper) in favor of a shorter course}


to minimize cost, side effects, and long-term complications.

\section{WHEN DO THE GUIDELINES NOT APPLY?}

Although these guidelines address decision-making for patients with symptomatic ITP with severe thrombocytopenia, there is limited guidance about treating asymptomatic patients whose platelet counts are below $100 \times 10^{9} / \mathrm{L}$ but over $30 \times 10^{9} / \mathrm{L}$. The guidelines are broadly applicable to ITP management and to most patient populations. However, the guidelines do not specifically comment on pregnant patients, management of secondary ITP, or treatment options beyond the use of TPO-RA, rituximab, or splenectomy as second-line agents. Fostamatinib, a splenic tyrosine-kinase inhibitor, is an approved ITP therapy but is not specifically discussed in these guidelines, as it has primarily been studied in the third-line setting.

\section{CONCLUSION}

The updated ASH guidelines are meant to help with clinical judgment and patient care, especially if multiple treatment options are available. Despite the guidelines, which include some direct recommendations, clinical judgment should prevail. Also, the guidelines may not always apply. For example, there is no concrete evidence that an asymptomatic patient with a normal bleeding risk and a platelet count just under $30 \times 10^{9} / \mathrm{L}$ will have a different meaningful outcome if treated with close observation vs corticosteroid treatment.

It is important to note that these guidelines are not exhaustive and do not serve as a substitute for discussions between providers and patients. These recommendations support shared decision-making as a method to individualize care based on the available options and patient preferences. When appropriate, clinical trial enrollment should be considered to help improve our knowledge and care of this patient population.

\section{DISCLOSURES}

Dr. DeSouza reports being an advisor or review panel participant for Sanofi. Dr. Angelini reports no relevant financial relationships which, in the context of her contributions, could be perceived as a potential conflict of interest.

3. Matzdorff A, Meyer O, Ostermann H, et al. Immune thrombocytopenia-current diagnostics and therapy: recommendations of a joint working group of DGHO, OGHO, SGH, GPOH, and DGTI. Oncol Res Treat 2018; 41(Suppl 5):1-30. doi:10.1159/000492187

Address: Dana Angelini, MD, Department of Hematology and Medical Oncology, CA-60, Cleveland Clinic, 9500 Euclid Avenue, Cleveland, OH 44195; angelid@ccf.org 